\title{
OPEN Acute liver injury following acetaminophen administration does not activate atrophic pathways in the mouse diaphragm
}

\author{
C. S. Bruells ${ }^{1,3 凶}$, P. Duschner ${ }^{1,3}$, G. Marx ${ }^{1}$, G. Gayan-Ramirez ${ }^{4}$, N. Frank ${ }^{1}$, T. Breuer ${ }^{1}$, \\ O. Krenkel ${ }^{2}$, F. Tacke ${ }^{5} \&$ J. C. Mossanen ${ }^{1}$
}

$\mathrm{N}$-acetyl-para-amino phenol (APAP, usually named paracetamol), which is commonly used for its analgesic and antipyretic properties may lead to hepatotoxicity and acute liver damage in case of overdoses. Released cytokines and oxidative stress following acute liver damage may affect other organs' function notably the diaphragm, which is particularly sensitive to oxidative stress and circulating cytokines. We addressed this issue in a mouse model of acute liver injury induced by administration of APAP. C57BL/6J mice (each $\mathrm{n}=8$ ) were treated with $\mathrm{N}$-acetyl-para-amino phenol (APAP) to induce acute drug caused liver injury and sacrificed 12 or $24 \mathrm{~h}$ afterwards. An untreated group served as controls. Key markers of inflammation, proteolysis, autophagy and oxidative stress were measured in diaphragm samples. In APAP treated animals, liver damage was proven by the enhanced serum levels of alanine aminotransferase and aspartate aminotransferase. In the diaphragm, besides a significant increase in IL 6 and lipid peroxidation, no changes were observed in key markers of the proteolytic, and autophagy signaling pathways, other inflammatory markers and fiber dimensions. The first $\mathbf{2 4} \mathrm{h}$ of acute liver damage did not impair diaphragm atrophic pathways although it slightly enhanced IL- 6 and lipid peroxidation. Whether longer exposure might affect the diaphragm needs to be addressed in future experiments.

Drug-induced liver injury represents a serious problem growing in prevalence and importance. Acute liver damage is induced by several toxic agents, of which $\mathrm{N}$-acetyl-para-amino phenol (APAP, usually named paraceta$\mathrm{mol}$ ) is the most common drug responsible for toxic liver injury ${ }^{1}$. APAP is highly used due to its analgesic and antipyretic properties and while safe and effective at therapeutic doses, its overdose may lead to hepatotoxicity and acute liver damage. At therapeutic doses, most of APAP is metabolized by phase II conjugating enzymes and excreted as a non-toxic compound. The remaining APAP is degraded via the cytochrome p-450 system into a more toxic substance, $\mathrm{N}$-acetyl-p-benzoquinone imine, whose toxic action can be prevented by glutathione $e^{1,2}$. In APAP overdose, glutathione scavenger reserves fade and, once depleted, the toxic metabolite damages liver cells, especially mitochondria where it leads to mitochondrial membrane permeabilization and dysfunction ${ }^{3}$.

Hence, acute liver injury can end as a systemic disease with decreasing liver synthesis and detoxification function and increasing cytokine production. Several authors pointed to the fact that acute liver injury is probably not an entity that remains limited to the liver ${ }^{4,5}$. In acute damage, the liver releases cytokines into the circulation ${ }^{4,5}$ and secondarily may influence other organs' function and biology.

The diaphragm is particularly sensitive to oxidative stress and circulating cytokines ${ }^{6,7}$ and diaphragm weakness with muscle atrophy through activation of several proteolytic pathways and autophagy has been reported in situations associated with enhanced circulating cytokines and/or oxidative stress ${ }^{8,9}$.

\footnotetext{
${ }^{1}$ Department of Intensive and Intermediate Care, University Hospital Aachen, Aachen, Germany. ${ }^{2}$ Department of Medicine III, University Hospital Aachen, Aachen, Germany. ${ }^{3}$ Department of Anesthesiology, University Hospital Aachen, Aachen, Germany. ${ }^{4}$ Laboratory of Pneumology, Katholieke Universiteit Leuven, Leuven, Belgium. ${ }^{5}$ Department of Hepatology \& Gastroenterology, Charité University Medical Center, Berlin, Germany. ${ }^{\varpi}$ email: cbruells@ukaachen.de
} 
We therefore hypothesized that acute liver injury may affect the diaphragm and induce atrophic signaling via protease-based proteolysis and autophagy. This issue was addressed in a mouse model of acute liver injury induced by administration of APAP.

\section{Methods}

The study was approved by the appropriate governmental institution (Landesamt für Natur-, Umwelt- und Verbraucherschutz, LANUV, Germany, reference number: AZ 84-02.04.2014.A165) which granted the ethical approval for this study. The study was conducted in accordance with the principles for care and use of animals based on the Helsinki Declaration and the ARRIVE criteria.

Induction of liver injury and animal model. C57BL/6J mice were housed under specific pathogen free conditions (Janvier labs, Le Genedt-Saint-Isle, France) and were divided into 3 groups (each n=8): (1) acute liver injury sacrified at $12 \mathrm{~h}$ (APAP12), (2) acute liver injury sacrified at $24 \mathrm{~h}$ (APAP24), (3) an untreated control group (CTRL). Acute liver injury was induced as described in detail before ${ }^{10,11}$. Briefly, after a fasting period of $12 \mathrm{~h}$, the animals received $250 \mathrm{mg} / \mathrm{kg}$ of APAP (Actavis Deutschland GmBh\& CO. Kg, Langenfeld, Germany) by a single i.v. injection into a tail vein. After sacrifice, diaphragm was removed quickly and further processed (see below). Alanine aminotransferase (ALT) and aspartate aminotransferase activity (UV test at $37^{\circ} \mathrm{C}$ ) was measured in serum (Roche modular preanalytics system; Roche Diagnostics International AG, Rotkreuz, Switzerland).

Diaphragm histology. Diaphragm strips were longitudinally embedded in formalin and cut in $10 \mu \mathrm{m}$ thin slides. Staining was performed using hematoxylin and eosin as routine laboratory staining. Around 50 fibers of 3 animals of each group were used for assessment of cross-sectional areas using Image J software (Image J, NIH, USA).

Protein extraction for electrophoresis and western blotting. Diaphragm tissue samples were homogenized 1:10 (weight volume ${ }^{-1}$ ) in $5 \mathrm{mmol}$ Tris. $\mathrm{HCl}$ and $5 \mathrm{mmol}$ EDTA buffer ( $\mathrm{pH}$ at 7.5) containing a protease inhibitor cocktail (Roche, Basel, Swiss) and centrifuged at $1500 \times g$ for $10 \mathrm{~min}$ at $4{ }^{\circ} \mathrm{C}$. After collection of the resulting supernatant, diaphragmatic protein content was assessed by the method of Bradford ${ }^{12}$, (Bio-Rad Laboratories Inc., Hercules, CA, USA). Proteins were detected with Chemiluminescent Peroxidase Substrate (Sigma-Aldrich, Overijse, Belgium), imaged with the Proxima 2850T imaging system (Isogen Life Technologies, De Meern, Netherlands) and analyzed using the TotalLab 1D software (Isogen Life Technologies, De Meern, Netherlands).

Autophagy and Pi3K-Akt pathway. Autophagosome formation was assessed by measuring the conversion of LC3B-I to LC3B-II via immunoblotting. Proteins were separated on a $12 \%$ polyacrylamide gel and transferred onto a polyvinylidene fluoride (PVDF) membrane. Blots were incubated overnight at $4{ }^{\circ} \mathrm{C}$ with the primary antibody (\# LC3A/B 4108S, Cell Signaling/Bioké, Leiden, The Netherlands) and subsequently with the appropriate secondary antibody (\# P0217, Agilent, Heverlee, Belgium) for $1 \mathrm{~h}$ at room temperature. Data were expressed as LC3B-II/LC3B-I ratio.

Upstream signaling via Phosphatidyl-inositol-Kinase-3 (Pi3K) and Protein kinase B (AKT) was also measured by detecting the phosphorylated and non-phosphorylated form of Pi3K (Pi3K; \#4249, Cell Signaling, Danvers USA) and of Protein kinase B (AKT; \#4691, Cell Signaling, Danvers USA). Data were expressed as ratio between phosphorylated to total (non-phosphorylated) protein.

Calpain-1 and caspase-3 activities. In vivo calpain-1 and caspase- 3 activities were indirectly assessed, by measuring cleavage of aII-spectrin, a specific substrate for both calpain-1 and caspase-3. After separation of the proteins on a $10 \%$ polyacrylamide gel and transfer to PVDF membrane, the blot was incubated with the primary antibody against $\alpha I I-s p e c t r i n$ ( $\alpha$-Fodrin FG6090, Enzo Life Science, Brussels, Belgium) and the suitable secondary antibody (P0260, Agilent, Heverlee, Belgium). Activities of calpain and caspase-3 were measured as the ratio between the densitometric values of their breakdown products to intact $\alpha$ II-spectrin ${ }^{13}$. The cleavage product of intact aII-spectrin by calpain gives a band at $150 \mathrm{kDa}$ and a band at $120 \mathrm{kDa}$ when cleaved by caspase- 3 while intact aII-spectrin is detected at $260 \mathrm{kDa}^{14}$. Active Caspase 3 was measured with their cleaved forms (Caspase-3: \#C9662, Cell Signaling, Danvers, USA).

Atrogin and MuRF-1. The activation of E3 ligases Atrogin and MURF-1 as markers of the ubiquitinproteasome pathway was investigated by Western blotting using the antibodies: (Atrogin: ECM Biosciences, \#AP2041, Versailles, USA; MURF-1: ABCAM, \#AB77577, Eugene; USA).

Oxidative stress: assessment of lipid peroxidation. Diaphragmatic 4-hydroxynonenal (4-HNE) was used as a marker of lipid peroxidation. After electrophoresis (7.5\% polyacrylamide gel and blotting) polyclonal anti-4-HNE antibody (\# MAB3249, R\&D Systems, Abingdon, UK) was used as primary antibody and a polyclonal rabbit anti-mouse (\# P0260, Agilent, Heverlee, Belgium) as secondary antibody. Data were expressed as a ratio between densitometric values of 4 -HNE and Vinculin ${ }^{15}$.

Inflammatory response: NF-KB subunits. The p50 and p 65 subunits of NFkB were assessed by Western blotting using P50/P65: \#4764 and phospho-P65: \#3031, Cell-Signaling, Danvers, MA, USA. 


\begin{tabular}{|l|l|}
\hline TNF-a for & CCACGTTGTAGCCAATGTC \\
\hline TNF-a rev & GAGTAGATGAGGTACAGCCC \\
\hline IL 6 for & GGTTCAATCAGGAGACCTGC \\
\hline IL 6 rev & GCTTTGTCTGGATTCTTTCCC \\
\hline IL-10 for & GCATCCACTTCCCAACCAG \\
\hline IL-10 rev & ACCCAGGTAACCCTTAAAGTC \\
\hline Casp3 for & TCATAATTCAGGCCTGCCGA \\
\hline Casp3 rev & GCTGCACAAAGTGACTGGA \\
\hline Casp7 for & CAT GGA TTT CCA GAA GAT GGG \\
\hline Casp7 rev & CTG TCA GAT CCT TTA TGG GTG \\
\hline IL-1ß1 for & TGAAGAAGAGCCCATCATCCT \\
\hline IL-1ß1 rev & TCATGCAGAACACCACTTCTC \\
\hline S7 for & AAATAAGCAGAAGCGTCCCA \\
\hline S7 rev & AATGTTTCAACCTTGTGCTCC \\
\hline
\end{tabular}

Table 1. Primers for mRNA detection. TNF tumor necrosis factor, $I L$ interleukin, Casp Caspase, $S 7$ ribosomal protein 7 .

RNA preparation and quantitative real-time PCR (qPCR). RNA from murine diaphragm samples were extracted with TRIZOL. $2 \mu \mathrm{g}$ purified RNA were reverse transcribed (Maxima H Minus Kit with dsDNase, Thermo Fisher \#K1682). qPCR was run on a StepOne Plus instrument (LifeTechnologies) using Power SYBR Green Master Mix (Applied Biosystem \#4368706). For messenger RNA (mRNA) analysis, inflammatory and proteolytic marker expression was normalized with ribosomal protein S7. RNA expression was analyzed by the $\Delta \Delta \mathrm{C}_{\mathrm{T}}$ method. The following primers were used (see Table 1).

Statistical analysis. Population distribution was assessed with the Kolmogorov-Smirnov test. If this test showed normal distribution of data, comparisons between groups for each dependent variable were made by ANOVA, if normal distribution was not present, by a Kruskall Wallis test. If the group effect was significant, a Dunn's multiple comparisons test was used for pairwise comparisons between all groups. Data are shown as means \pm standard deviation (SD). All statistical tests are two-tailed, significance was established at $\mathrm{p}<0.05$ (GraphPad Prism 6.0, La Jolla, CA, USA).

\section{Results}

Liver injury characteristics. Serum levels of ALT increased significantly and similarly 12 and $24 \mathrm{~h}$ after APAP administration compared to control (ctrl vs APAP $12 \mathrm{p}=0.01$; ctrl vs APAP $\mathrm{p}<0.001$ ) while serum levels of AST increased significantly but only $24 \mathrm{~h}$ after APAP administration (ctrl vs APAP $24 \mathrm{p}<0.001$ ) (see Fig. 1).

Histologic assessment of diaphragm atrophy. No differences in muscle fiber size could be detected between the experimental groups (see histogram as Fig. S1 in the Supplemental File 1).

Autophagy and Pi3K and AKT phosphorylation. The amount of Pi3K was not significantly changed 12 or $24 \mathrm{~h}$ after treatment (see Fig. 2 upper panel). Downstream, p-AKT/AKT was also not alter by intervention (see Fig. 2 lower panel). There were no changes in downstream conversion of LC3B-I to LC3B-II (see Fig. 3). See corresponding blots in Supplemental File 1, Figs. S6 and S10 as well as in Supplemental File 2, Figs. S1 and S6.

Proteolytic activation. Expression of Caspase 3 mRNA and Caspase 7 mRNA was not altered after 12 or $24 \mathrm{~h}$ compared to untreated controls. The protein levels of Pro-Caspase 3/Caspase 3 did not change at any time points compared to controls (mRNA data see Supplemental Fig. S3).

Caspase 3 and calpain-1 protein (measured as the amount of the specific breakdown products of $\alpha$-II spectrin) were significantly decreased after $24 \mathrm{~h}$ after APAP intoxication vs ctrl (caspase $3:-45 \%, \mathrm{p}=0.005$; calpain 1: $-45 \%, p=0.02$ ) (see Fig. S4 in Supplement 1, as well as the corresponding blots in Supplemental File 1, Fig. S7, Supplemental File 2, Fig. S2).

Activation of the Ubiquitin proteasome system. Downstream levels of Ubiquitin E3 ligases Atrogin and MURF did not differ between ctrl and the APAP treated animals (Fig. S5 in Supplement, as well as the corresponding blots in Supplemental File 1, Fig. S7, Supplemental File 2, Fig. S3).

Diaphragm inflammatory response. Levels of the NfkB subunits p50 and p65 were not altered in the different groups (see Fig. S6 in Supplement, as well as the corresponding blots in Supplemental File 1, Fig. S8 and Supplemental File 2, Fig. S4). The mRNA expression of IL-10, TNF- $\alpha$, IL1- $\beta$ did not change significantly while mRNA levels of IL-6 were significantly elevated $24 \mathrm{~h}$ after APAP exposure vs $\mathrm{ctrl}(\mathrm{p}=0.02)$ (see Fig. 4). 

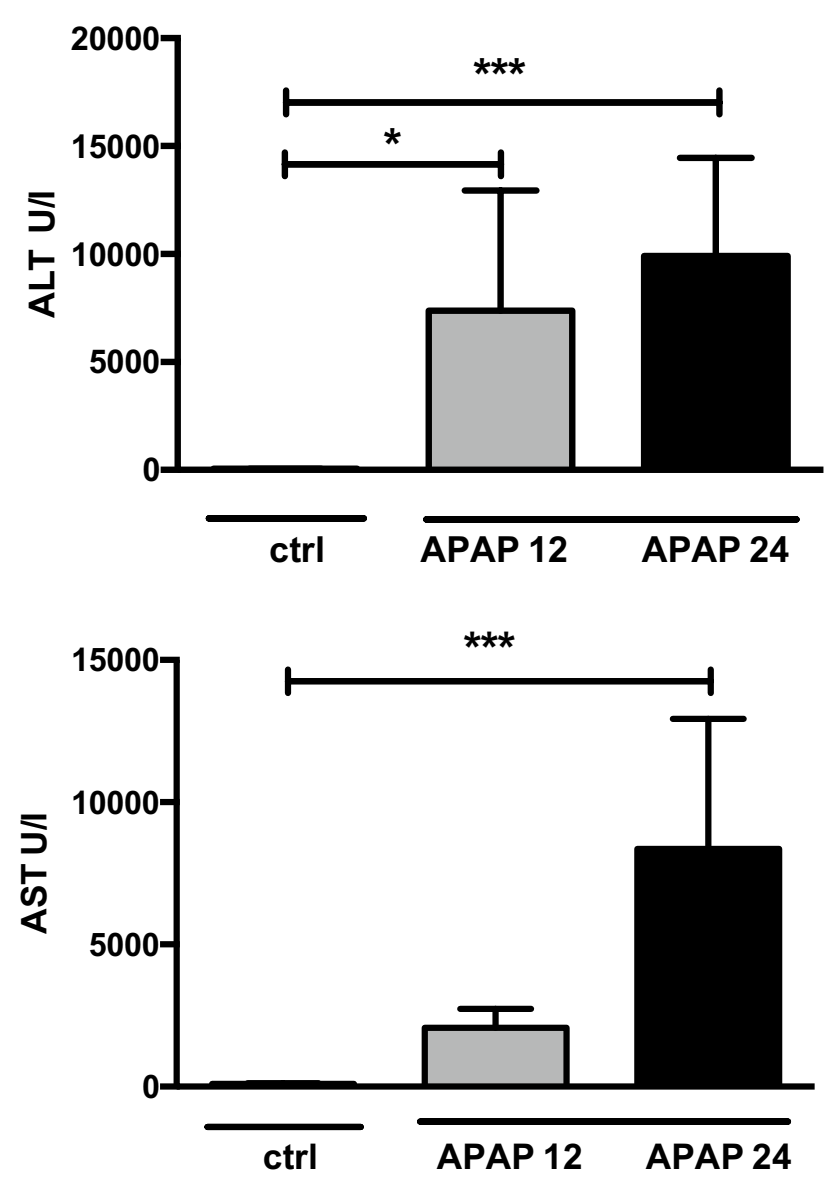

Figure 1. Serum levels of liver enzymes: alanine amino-transferase (ALT, upper panel) and aspartate aminotransferase activity (AST, lower panel) $12 \mathrm{~h}$ (APAP12) and $24 \mathrm{~h}$ (APAP24) after administration of $\mathrm{N}$-acetyl-para-amino phenol (APAP) compared to controls (ctrl). The increase in liver enzymes indicated severe liver injury during the experiment. Values are mean and standard deviation expressed in U/l. ${ }^{\star}$ and ${ }^{\star * *}$ indicate significance $\mathrm{p}<0.001$ or $\mathrm{p}<0.0001$ respectively.

Oxidative stress. Enhanced lipid peroxidation was present in the diaphragm $12 \mathrm{~h}$ after APAP exposure compared to controls $(+30 \%, \mathrm{p}=0.02)$, while this increase failed to reach statistical significance after $24 \mathrm{~h}$ (see Fig. 5). The blots are displayed in Supplemental File 1, Fig. S9 and Supplemental File 2, Fig. S5.

\section{Discussion}

This study showed that in mice, during the acute phase of liver injury, no activation of the proteolytic or autophagic pathways and no atrophy occurred in the diaphragm while lipid peroxidation was elevated and IL-6 was the only inflammatory marker to be upregulated inside the diaphragm. The effects on the diaphragm are discussed in detail below.

The APAP model to induce acute liver failure is a well-established model. In previous studies, we have proven that induction of necrosis in hepatocytes after administration of APAP leads to consistent results within groups. Analysis of immune cells and their modulation showed that the model induces "two hits" during acute liver injury generated by $\mathrm{APAP}^{10}$. Hepatocyte necroses are induced directly by the toxic metabolite NAPQI. Otherwise, severity of the liver injury and dimension of necrosis are affected by a massive immune cell infiltration into the liver caused by the initial damage ${ }^{3}$. Until know it is still ambiguous which part of the immune response and which immune cells respectively aggravate or attenuate liver injury at the different stages of the disease ${ }^{16}$.

We detected significant changes of liver enzymes' serum levels and necrotic areas inside liver tissue, pointing to a profound damage of hepatocytes. Central clinical signs of the acute liver failure are prolonged prothrombin time/INR, declined mental function, vasodilation and a systemic inflammatory response syndrome due to the high immunological impact of the liver ${ }^{4}$. Summarily, acute liver failure results in multi-organ failure (Acute liver failure $)^{17}$, although depending on etiology and duration course of illness is variable. In APAP and ischemia induced ALF the acute phase will be measured in hours and we expect systemic effects during the experimental time chosen in our model.

Inflammatory response to acute liver injury has been described in humans and several cytokines in drug induced liver injury were elevated in serum ${ }^{18}$. Blazka and colleagues revealed an immediate response of TNFa and IL1 $\beta$ after APAP induced liver injury induction with systemic response ${ }^{19}$. IL- 6 can even more act in several 

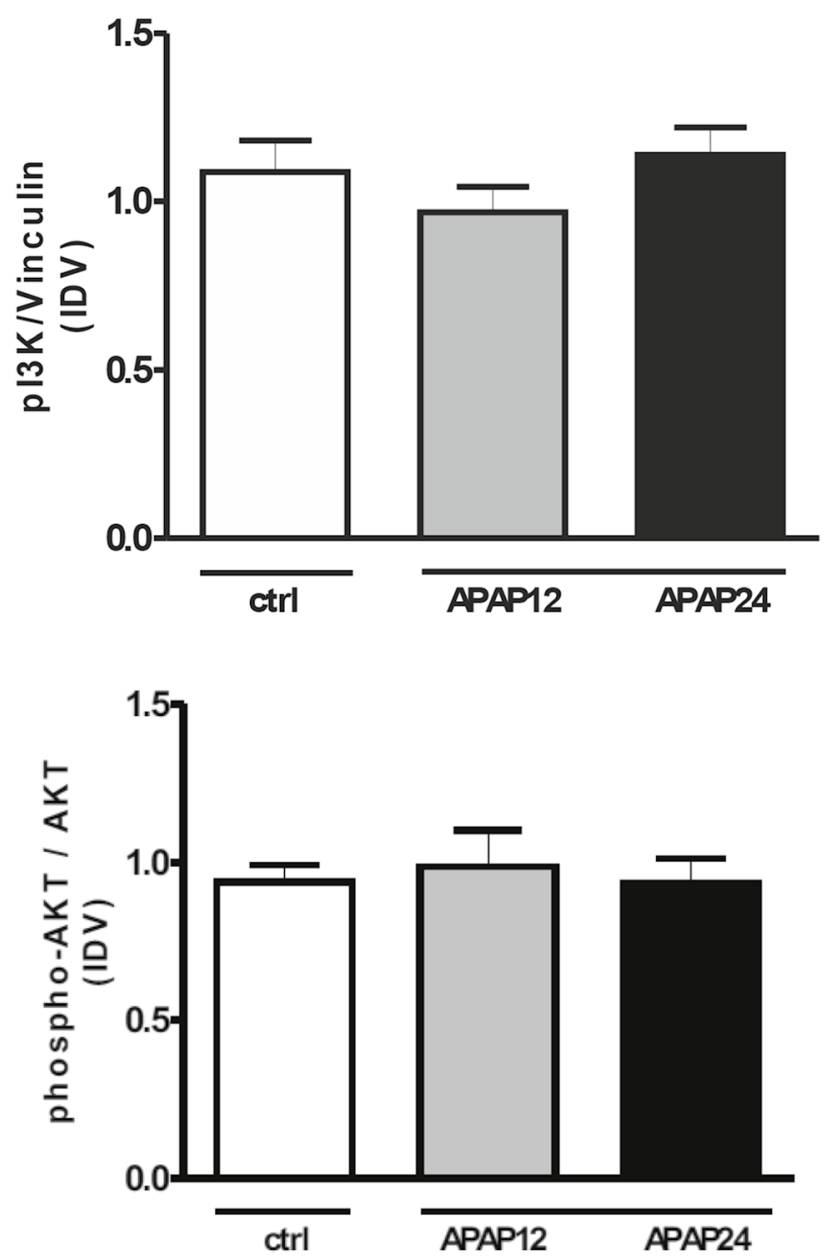

Figure 2. Changes in upstream signaling of protein synthesis. Upper panel Amount of Pi3K 12 or $24 \mathrm{~h}$ after APAP treatment. Lower panel changes of phosphorylated AKT vs AKT 12 or $24 \mathrm{~h}$ after APAP treatment. $P i 3 K$ phosphatidyl-inositol Kinase 3; AKT Protein kinase B; there is no change in these parameters. APAP $12 \mathrm{~N}$-acetyl-para-amino phenol $12 \mathrm{~h}$ treatment; APAP $24 \mathrm{~N}$-acetyl-para-amino phenol $24 \mathrm{~h}$ treatment, $c t r l$ controls, IDV integrated density value. Values are mean and standard deviation.

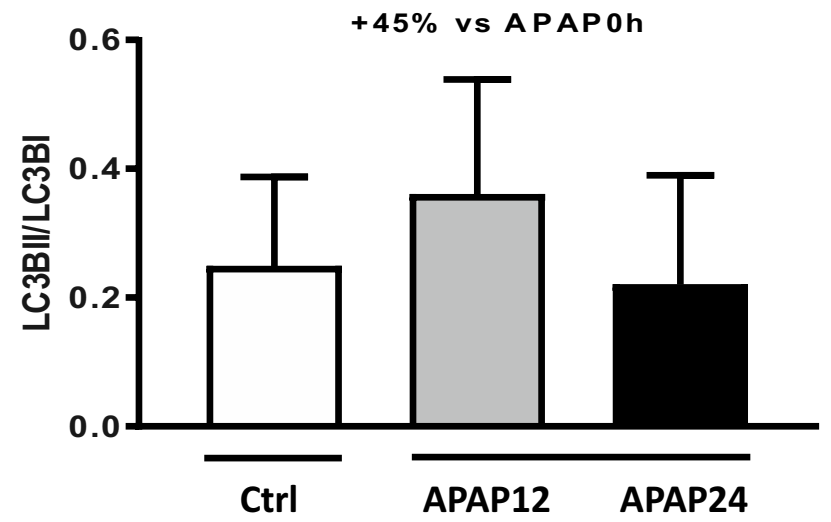

Figure 3. LC3B-I to LC3B-II ratio in control animals and after treatment. There is an increase in autophagosome formation after $12 \mathrm{~h}$, but this increase fails to reach statistical significance. APAP $12 \mathrm{~N}$-acetylpara-amino phenol $12 \mathrm{~h}$ treatment, APAP $24 \mathrm{~N}$-acetyl-para-amino phenol $24 \mathrm{~h}$ treatment, ctrl controls; Values are mean and standard deviation.

different ways in muscle and is produced during exercise ${ }^{20}$ or diaphragm overload ${ }^{21}$. Moreover, Janssen et al. 

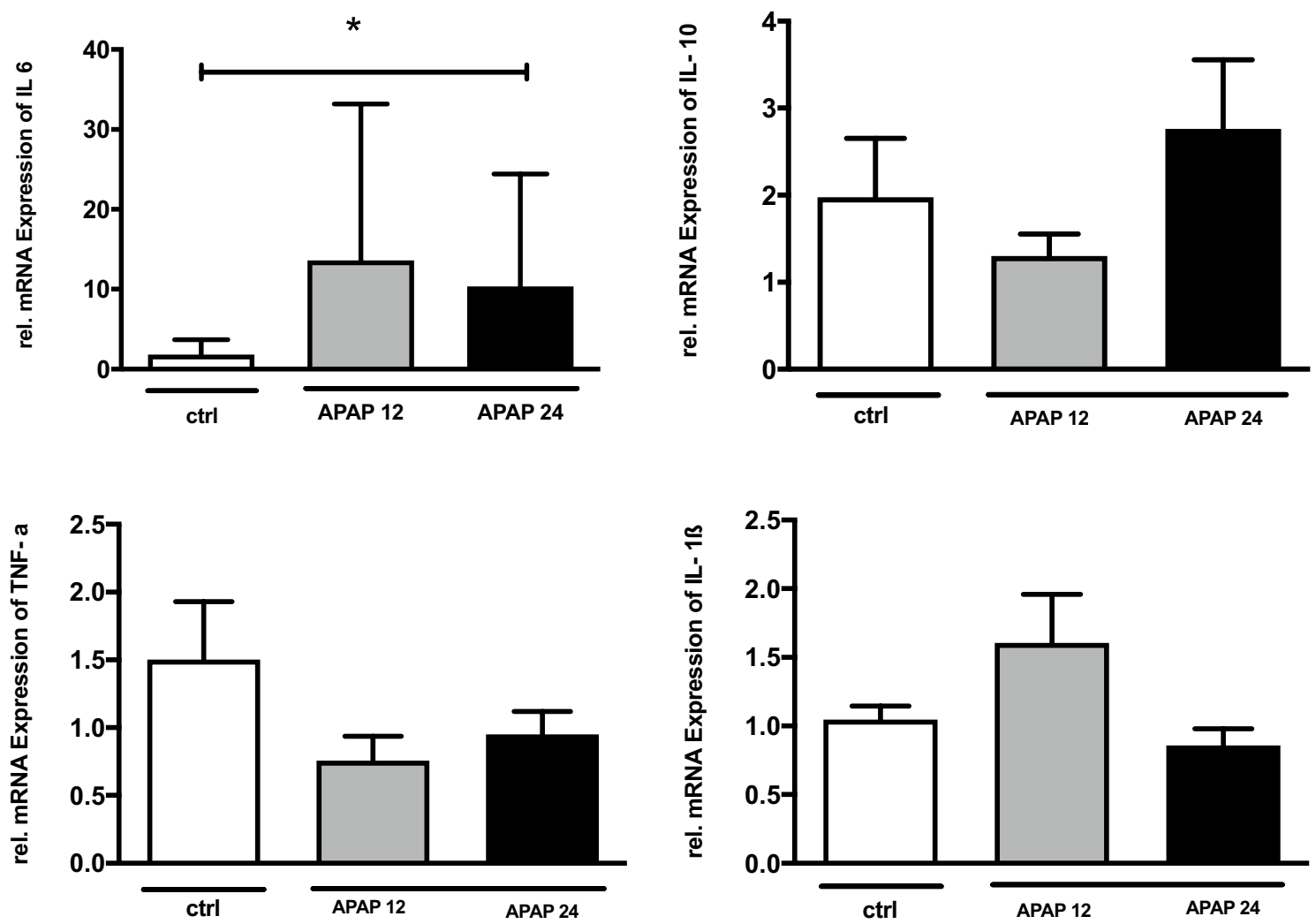

Figure 4. Inflammatory response in diaphragm tissue. IL-6, IL-10, TNFalpha and IL-1b mRNA expression relative to ctrl demonstrating a significant increase in IL-6 after APAP $12 \mathrm{~N}$-acetyl-para-amino phenol $12 \mathrm{~h}$ treatment; APAP $24 \mathrm{~N}$-acetyl-para-amino phenol $24 \mathrm{~h}$ treatment compared to control (ctrl). Values are mean and standard deviation ${ }^{*} \mathrm{p}<0.02$.

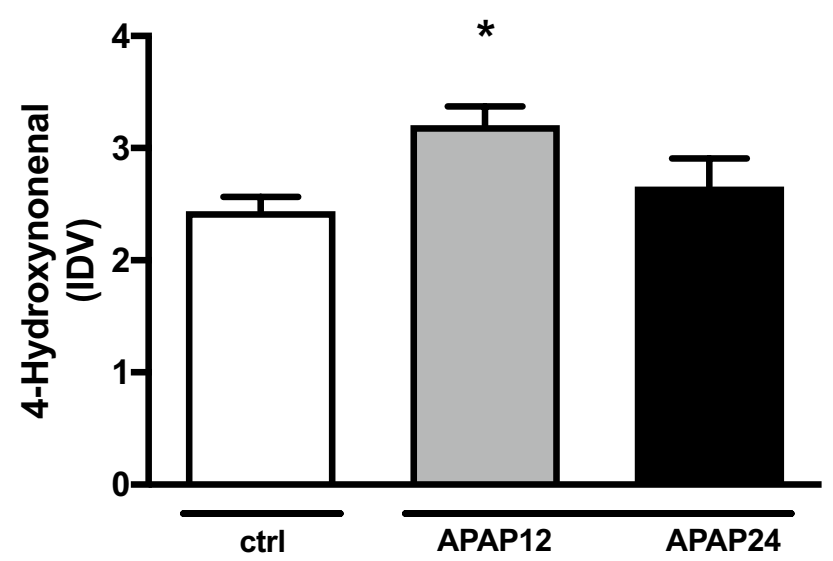

Figure 5. Lipid peroxidation in diaphragm tissue in interventional groups and control animals. $12 \mathrm{~h}$ after APAP exposure, there is a significant increase in lipid peroxidation. 4-HNE 4-Hydroxdy-non-enal, APAP $12 \mathrm{~N}$-acetylpara-amino phenol $12 \mathrm{~h}$ treatment, APAP $24 \mathrm{~N}$-acetyl-para-amino phenol $24 \mathrm{~h}$ treatment, $c t r l$ controls; Data are normalized by vinculin and expressed as mean and SD. IDV integrated density value; ${ }^{*}$ indicates significance $\mathrm{p}<0.05$.

demonstrated severe muscle atrophy in the diaphragm after IL-6 administration into the circulation. However, in diaphragm the influence of inflammation on its function has been under discussion: Blocking of toll-like receptor- 4 in mice in a disuse model could decrease the amount of autophagic activation and IL- 6 production and ameliorate the reduction of myosin heavy chain protein ${ }^{22}$. Possibly, the animals had an increase in the work of breathing due to metabolic disturbances after liver injury, which might explain this increase in IL-6 in a quasiphysiologic response to overload. 
Summarily, we could not detect a profound secondary response inside the diaphragm in the following $12 \mathrm{~h}$ or $24 \mathrm{~h}$ after liver injury induction except for enhanced levels of IL-6 that were not associated with changes in NFKB subunits or other inflammatory cytokines.

Oxidative stress is a key factor in the pathogenesis of ICU-acquired diaphragmatic weakness ${ }^{5}$ with mitochondria and to a smaller extent oxidases functioning as major sources of $\operatorname{ROS}^{23-25}$. In diaphragm, the increase of ROS interacts with the activity of main proteolytic pathways caspase 3 and calpain- 1 as well as the autophagic system, so that blocking ROS results in an amelioration of atrophy via autophagic and proteolytic pathways ${ }^{23,24,26,27}$. In our model, we could detect a significant transient increase in the formation of lipid peroxidation $(4 \mathrm{HNE})$ after $12 \mathrm{~h}$ of APAP induced liver injury. This increase abandoned in the $24 \mathrm{~h}$ APAP group, which could indicate a quick removal of oxygenated structures, as we have detected in a former experiment ${ }^{28}$. In our actual study, we did not distinguish further which source (mitochondria and/or oxidases) was responsible for the increase in oxidation. Importantly, we did not found a link between this increase in lipid peroxiadation and atrophy inducing pathways, although in disuse or sepsis this has been proven ${ }^{26,27}$. Possibly the amount of oxidative stress did not reach the level to induce proteolytic Ca-dependent proteases or autophagy.

In several diseases, proteolysis is activated inside the diaphragm and modulated by different pathways: Calcium dependent protease-based proteolysis via caspase 3 and calpain 1 have been described during sepsis, disuse (VIDD) and in chronic lung disease ${ }^{29-31}$. Caspase 3 acts a key protease, in conjunction with calpain 1 , to break up the sarcomeric structures and allow downstream proteasome to further degrade the proteins ${ }^{32,33}$. Neither mRNA levels nor protein expression of key calcium-dependent proteases were enhanced in the diaphragm despite the presence of clear liver injury. Even more, the downstream ligases MURF and MAFbx remained unaltered in the interventional groups, although these ligases are involved in the further degradation of muscle protein after break-up by caspases ${ }^{31}$ and are usually activated during muscle atrophy, inflammation or oxidative stress ${ }^{30}$. In summary, proteolytic activation in the diaphragm by acute liver injury does not seem to be present in our model.

Autophagy is an important proteolytic pathway targeting cellular components associated with muscular atrophy via autophagosomes, which form double layer structures of which LC3 is a core molecule ${ }^{27,34}$. Upstream, Protein kinase B (AKT) and PI3K regulate via the Forkhead-Box-O1 transcription factor the formation of autophagosome building proteins, like LC3B.

The diaphragm is susceptible to slightest disturbances in the ratio of protein synthesis and proteolysis, so that even $12 \mathrm{~h}$ of disuse affect this balance ${ }^{35}$. Transformation from LCB3BI into LC3B-II indicates the activation of autophagosomes ${ }^{30}$ and Hussain and colleagues described this pathway as one major diaphragmatic pathway in disuse $\mathrm{s}^{36}$ which is also active in sepsis and linked to oxidative stress ${ }^{30}$. In addition, the autophagy pathway can modulate protein synthesis through the mammalian target of rapamycin (mTOR) and serves therefore as a switch between anabolic and catabolic states ${ }^{37}$.

During liver injury as in our model, autophagy assessed by the ratio of LC3BII/LC3BI and by the upstream molecules was not activated in the diaphragm.

This study has, however, some limitations that should be taken into account. According to the goal of the study, 12 and 24 h were specifically chosen as time point measures after liver injury in order to address the early phase effects of liver injury on the diaphragm. We, however, acknowledge that later time point measures may be relevant to address the secondary complications reported to arise days after acute liver injury in humans. Further studies are needed to address the influence of acetaminophen induced liver injury on diaphragm dysfunction at later time points and whether known effects on lung function (e.g. airway hyper-reactivity, asthma, etc.) may add to these changes ${ }^{38}$. Those were in this study beyond the scope.

\section{Conclusion}

In contrast to our hypothesis, acute liver injury induced by APAP in mouse did not induce atrophy or activate proteolysis via calcium dependent proteases or via ubiquitin proteasome pathway, neither it did stimulate autophagy. However, it resulted in enhanced diaphragm lipid peroxidation and upregulation of IL-6 which, on long-term, might be detrimental for the diaphragm. Future research might focus on long term models of liver injury to see whether the diaphragm would be affected.

Received: 9 January 2020; Accepted: 4 March 2021

Published online: 18 March 2021

\section{References}

1. Krenkel, O., Mossanen, J. C. \& Tacke, F. Immune mechanisms in acetaminophen-induced acute liver failure. Hepatobiliary Surg. Nutr. 3, 331-343. https://doi.org/10.3978/j.issn.2304-3881.2014.11.01 (2014).

2. James, L. P., Mayeux, P. R. \& Hinson, J. A. Acetaminophen-induced hepatotoxicity. Drug Metab. Dispos. Biol. Fate Chem. 31, 1499-1506. https://doi.org/10.1124/dmd.31.12.1499 (2003).

3. Yan, M., Huo, Y., Yin, S. \& Hu, H. Mechanisms of acetaminophen-induced liver injury and its implications for therapeutic interventions. Redox Biol. 17, 274-283. https://doi.org/10.1016/j.redox.2018.04.019 (2018).

4. Bernal, W., Lee, W. M., Wendon, J., Larsen, F. S. \& Williams, R. Acute liver failure: A curable disease by 2024?. J. Hepatol. 62, S112120. https://doi.org/10.1016/j.jhep.2014.12.016 (2015).

5. Powers, S. K., Kavazis, A. N. \& Deruisseau, K. C. Mechanisms of disuse muscle atrophy: Role of oxidative stress. Am. J. Physiol. Regul. Integr. Comp. Physiol. 288, R337-R344 (2005).

6. Janssen, S. P. et al. Interleukin-6 causes myocardial failure and skeletal muscle atrophy in rats. Circulation 111, 996-1005. https:// doi.org/10.1161/01.CIR.0000156469.96135.0D (2005). 
7. McClung, J. M. et al. Antioxidant administration attenuates mechanical ventilation-induced rat diaphragm muscle atrophy independent of protein kinase B (PKB Akt) signalling. J. Physiol. 585, 203-215 (2007).

8. Callahan, L. A. \& Supinski, G. S. Sepsis induces diaphragm electron transport chain dysfunction and protein depletion. Am. J. Respir. Crit. Care Med. 172, 861-868. https://doi.org/10.1164/rccm.200410-1344OC (2005).

9. Jung, B. et al. Diaphragmatic dysfunction in patients with ICU-acquired weakness and its impact on extubation failure. Intens. Care Med. 42, 853-861. https://doi.org/10.1007/s00134-015-4125-2 (2016).

10. Mossanen, J. C. et al. Chemokine (C-C motif) receptor 2-positive monocytes aggravate the early phase of acetaminophen-induced acute liver injury. Hepatology 64, 1667-1682. https://doi.org/10.1002/hep.28682 (2016).

11. Mossanen, J. C. \& Tacke, F. Acetaminophen-induced acute liver injury in mice. Lab. Anim. 49, 30-36. https://doi.org/10.1177/ $0023677215570992(2015)$.

12. Bradford, M. M. A rapid and sensitive method for the quantitation of microgram quantities of protein utilizing the principle of protein-dye binding. Anal. Biochem. 72, 248-254 (1976).

13. Bruells, C. S. et al. Sedation using propofol induces similar diaphragm dysfunction and atrophy during spontaneous breathing and mechanical ventilation in rats. Anesthesiology 120, 665-672. https://doi.org/10.1097/ALN.0000000000000125 (2014).

14. Agten, A. et al. Bortezomib partially protects the rat diaphragm from ventilator-induced diaphragm dysfunction. Crit. Care Med. 40, 2449-2455. https://doi.org/10.1097/CCM.0b013e3182553a88 (2012).

15. Bruells, C. S. et al. Influence of weaning methods on the diaphragm after mechanical ventilation in a rat model. BMC Pulm. Med. 16, 127 (2016).

16. Woolbright, B. L. \& Jaeschke, H. Role of the inflammasome in acetaminophen-induced liver injury and acute liver failure. J. Hepatol. 66, 836-848. https://doi.org/10.1016/j.jhep.2016.11.017 (2017).

17. Bernal, W. \& Wendon, J. Acute liver failure. N. Engl. J. Med. 369, 2525-2534. https://doi.org/10.1056/NEJMra1208937 (2013).

18. Steuerwald, N. M. et al. Profiles of serum cytokines in acute drug-induced liver injury and their prognostic significance. PLoS ONE 8, e81974. https://doi.org/10.1371/journal.pone.0081974 (2013).

19. Blazka, M. E., Wilmer, J. L., Holladay, S. D., Wilson, R. E. \& Luster, M. I. Role of proinflammatory cytokines in acetaminophen hepatotoxicity. Toxicol. Appl. Pharmacol. 133, 43-52. https://doi.org/10.1006/taap.1995.1125 (1995).

20. Pedersen, B. K., Steensberg, A. \& Schjerling, P. Muscle-derived interleukin-6: Possible biological effects. J. Physiol. 536, 329-337. https://doi.org/10.1111/j.1469-7793.2001.0329c.xd (2001).

21. Sigala, I. et al. Nitric oxide regulates cytokine induction in the diaphragm in response to inspiratory resistive breathing. J. Appl. Physiol. 113, 1594 (2012)

22. Schellekens, W. J. et al. Toll-like receptor 4 signaling in ventilator-induced diaphragm atrophy. Anesthesiology 117, 329-338. https:// doi.org/10.1097/ALN.0b013e3182608cc0 (2012).

23. Powers, S. K. et al. Mitochondria-targeted antioxidants protect against mechanical ventilation-induced diaphragm weakness. Crit. Care Med. 39, 1749-1759 (2011).

24. Picard, M. et al. Mitochondrial dysfunction and lipid accumulation in the human diaphragm during mechanical ventilation. Am. J. Respir. Crit. Care Med. 186, 1140-1149. https://doi.org/10.1164/rccm.201206-0982OC (2012).

25. Whidden, M. A. et al. Xanthine oxidase contributes to mechanical ventilation-induced diaphragmatic oxidative stress and contractile dysfunction. J. Appl. Physiol. 106, 385-394 (2009).

26. Whidden, M. A. et al. Oxidative stress is required for mechanical ventilation-induced protease activation in the diaphragm. J. Appl. Physiol. 108, 1376-1382 (2010).

27. Smuder, A. J. et al. Crosstalk between autophagy and oxidative stress regulates proteolysis in the diaphragm during mechanical ventilation. Free Radic. Biol. Med. 115, 179-190. https://doi.org/10.1016/j.freeradbiomed.2017.11.025 (2018).

28. Bruells, C. S., Rossaint, R., Du, J., Bleilevens, C., Goetzenich, A., Weis, J., Wiggs, M. P., Powers, S. K. \& Hein, M. Diaphragmatic repair after onset of ventilator induced diaphragmatic dysfunction needs longer than ventilation time. In Congress of the American Thoracic Society Philadelphia May 17-22, A4834 (2012).

29. McClung, J. M., Judge, A. R., Talbert, E. E. \& Powers, S. K. Calpain-1 is required for hydrogen peroxide-induced myotube atrophy. Am. J. Physiol. Cell Physiol. 296, C363-C371 (2009).

30. Maes, K. et al. Effects of controlled mechanical ventilation on sepsis-induced diaphragm dysfunction in rats. Crit. Care Med. 42, e772-e782. https://doi.org/10.1097/ccm.0000000000000685 (2014).

31. van Hees, H. et al. Proteasome inhibition improves diaphragm function in an animal model for COPD. Am. J. Physiol. Lung Cell Mol. Physiol. 301, L110-L116. https://doi.org/10.1152/ajplung.00396.2010 (2011).

32. Nelson, W. B., Smuder, A. J., Hudson, M. B., Talbert, E. E. \& Powers, S. K. Cross-talk between the calpain and caspase-3 proteolytic systems in the diaphragm during prolonged mechanical ventilation. Crit. Care Med. 40, 1857-1863. https://doi.org/10.1097/CCM. 0b013e318246bb5d (2012).

33. Smuder, A. J., Nelson, W. B., Hudson, M. B., Kavazis, A. N. \& Powers, S. K. Inhibition of the ubiquitin-proteasome pathway does not protect against ventilator-induced accelerated proteolysis or atrophy in the diaphragm. Anesthesiology 121, 115-126. https:// doi.org/10.1097/aln.0000000000000245 (2014).

34. Tang, H. et al. Intrinsic apoptosis in mechanically ventilated human diaphragm: Linkage to a novel Fos/FoxO1/Stat3-Bim axis. FASEB J. 25, 2921-2936. https://doi.org/10.1096/fi.11-183798 (2011).

35. Shanely, R. A. et al. Mechanical ventilation depresses protein synthesis in the rat diaphragm. Am. J. Resp. Crit. Care Med. 170, 994-999 (2004).

36. Hussain, S. N. A. et al. Mechanical ventilation-induced diaphragm disuse in humans triggers autophagy. Am. J. Resp. Crit. Care Med. 182, 1377-1386 (2010).

37. Schiaffino, S. \& Mammucari, C. Regulation of skeletal muscle growth by the IGF1-Akt/PKB pathway: Insights from genetic models. Skelet. Muscle 1, 4. https://doi.org/10.1186/2044-5040-1-4 (2011).

38. Kennon-McGill, S. \& McGill, M. R. Extrahepatic toxicity of acetaminophen: Critical evaluation of the evidence and proposed mechanisms. J. Clin. Transl. Res. 3, 297-310 (2018).

\section{Author contributions}

C.B. and J.C.M. designed the experiment; wrote manuscript; statistics. P.D. Western blots, PCR, animal experiment, statistics. G.GR Western blots. T.B. statistics, manuscript writing. O.K. animal experiment. F.T. animal experiment, experimental design, data acquisition, manuscript. N.F. Western blots, PCR. G.M. Manuscript writing.

\section{Funding}

Open Access funding enabled and organized by Projekt DEAL.

\section{Competing interests}

The authors declare no competing interests. 


\section{Additional information}

Supplementary Information The online version contains supplementary material available at https://doi.org/ 10.1038/s41598-021-85859-2.

Correspondence and requests for materials should be addressed to C.S.B.

Reprints and permissions information is available at www.nature.com/reprints.

Publisher's note Springer Nature remains neutral with regard to jurisdictional claims in published maps and institutional affiliations.

(c) (i) Open Access This article is licensed under a Creative Commons Attribution 4.0 International License, which permits use, sharing, adaptation, distribution and reproduction in any medium or format, as long as you give appropriate credit to the original author(s) and the source, provide a link to the Creative Commons licence, and indicate if changes were made. The images or other third party material in this article are included in the article's Creative Commons licence, unless indicated otherwise in a credit line to the material. If material is not included in the article's Creative Commons licence and your intended use is not permitted by statutory regulation or exceeds the permitted use, you will need to obtain permission directly from the copyright holder. To view a copy of this licence, visit http://creativecommons.org/licenses/by/4.0/.

(C) The Author(s) 2021 